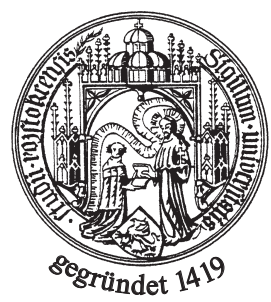

17

\author{
Aurelia Merlan / Jürgen Schmidt-Radefeldt \\ (Hrsg./eds.)
}

\title{
Portugiesisch als Diasystem O Português como Diassistema
}

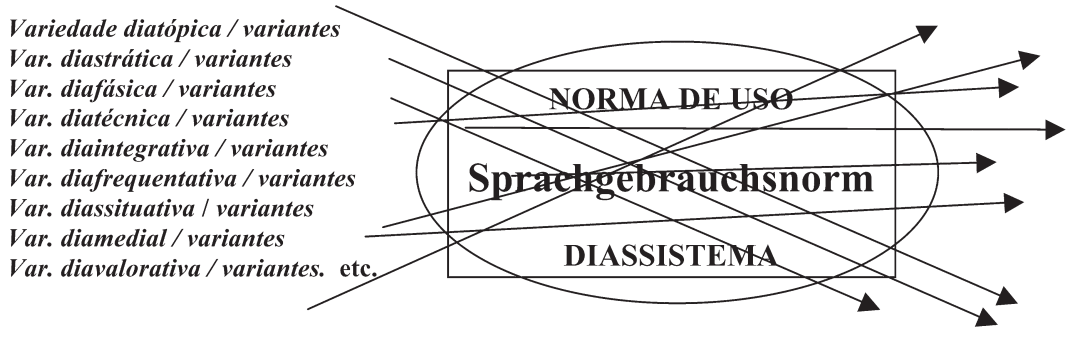


Das Diasystem des Portugiesischen umfasst einerseits nationale Varietäten, andererseits Minderheitenvarietäten. Jede nationale Varietät für sich stellt ihrerseits ein Diasystem dar: Es gibt sprachliche Unterschiede im Raum, in der sozialen Schichtung, in der Ausdrucksweise, zwischen Berufsgruppen, zwischen Generationen usw. Die Beiträge dieses Bandes haben das Ziel, in Einzelanalysen Teilbereiche der verschiedensten Varietäten des Portugiesischen auszubreiten, Spezifika möglichst aufgrund corpus-basierter Dateien oder Einzelbeobachtungen darzustellen und auch den Forschungsstand der jeweilig dominanten diasystematischen Teilbereiche zu berücksichtigen.

O diassistema do Português abrange variedades nacionais assim como variedades minoritárias. Cada variedade nacional per se representa um diassistema: existem diferenças linguísticas no espaço, nos estratos sociais, no modo de expressão, entre grupos profissionais, entre gerações, etc. O objectivo dos artigos contidos neste volumen consiste em analisar, em estudos individuais, certas áreas das mais diversas variedades do Português e apresentar particularidades específicas o mais possível com base em corpora linguísticos ou em observações individuais, tomando também em consideração o estado actual da investigação nos domínios diassistemáticos dominantes.

Aurelia Merlan lehrt als Privatdozentin Romanische Sprachwissenschaft an der Ludwig-Maximilians-Universität München.

Jürgen Schmidt-Radefeldt ist Professor emeritus für Romanische Sprachwissenschaft an der Universität Rostock.

Aurélia Merlan ensina Linguística Românica na Universidade Ludwig Maximilian de Munique.

Jürgen Schmidt Radefeldt é professor emérito de Linguística Românica na Universidade de Rostock. 
Portugiesisch als Diasystem

O Português como Diassistema 


\section{ROSTOCKER ROMANISTISCHE ARBEITEN}

Herausgegeben von

Aurelia Merlan, Jürgen Schmidt-Radefeldt und Rudolf Windisch

Band 17

PETER LANG 


\section{Aurelia Merlan / Jürgen Schmidt-Radefeldt \\ (Hrsg./eds.)}

\section{Portugiesisch als Diasystem \\ O Português como Diassistema}




\section{Bibliografische Information der Deutschen Nationalbibliothek}

Die Deutsche Nationalbibliothek verzeichnet diese Publikation in der Deutschen Nationalbibliografie; detaillierte bibliografische Daten sind im Internet über http://dnb.d-nb.de abrufbar.

Die Veröffentlichung dieses Buches wurde unterstützt durch Fundação Calouste Gulbenkian, Lisboa.

\section{FUNDAÇÃO CALOUSTE GULBENKIAN}

Abbildung auf dem Umschlag:

Sigillum universitatis studii rostokcensis, gegründet 1419.

Abdruck mit freundlicher Genehmigung

des Rektors der Universität Rostock.

Gedruckt auf alterungsbeständigem, säurefreiem Papier.

ISSN $1437-3130$

ISBN 978-3-631-64109-5 (Print)

E-ISBN 978-3-653-02802-7 (E-Book)

DOI 10.3726/978-3-653-02802-7

(C) Peter Lang GmbH

Internationaler Verlag der Wissenschaften

Frankfurt am Main 2013

Alle Rechte vorbehalten.

Peter Lang Edition ist ein Imprint der Peter Lang GmbH.

Peter Lang - Frankfurt am Main · Bern · Bruxelles · New York ·

Oxford $\cdot$ Warszawa $\cdot$ Wien

Das Werk einschließlich aller seiner Teile ist urheberrechtlich geschützt. Jede Verwertung außerhalb der engen Grenzen des

Urheberrechtsgesetzes ist ohne Zustimmung des Verlages unzulässig und strafbar. Das gilt insbesondere für

Vervielfältigungen, Übersetzungen, Mikroverfilmungen und die Einspeicherung und Verarbeitung in elektronischen Systemen.

Dieses Buch erscheint in der Peter Lang Edition und wurde vor Erscheinen peer reviewed.

www.peterlang.com 


\section{Rostocker Romanistische Arbeiten}

Herausgegeben von Aurelia Merlan, Jürgen Schmidt-Radefeldt und Rudolf Windisch

Band 1 Renate Büscher-Grotehusmann: Maya-K'iche' und Spanisch - Sprachkontakt und Sprachkonflikt in Guatemala. Eine soziolinguistische Beschreibung der Comunidad de Zunil. 1999.

Band 2 Anett Heil: Grammatische Reduktion in Frankokreolsprachen und Plansprachen. 1999.

Band 3 Hans-Burkard Krause: Lexikologische Beschreibungen zum konzeptuell-semantischen Netz intelligence im heutigen Französisch. 1999.

Band 4 Karin Weise: Kontrastive Textanalyse zu einigen ausgewählten Vergangenheitstempora des Portugiesischen und des Deutschen. 2000.

Band 5 Huldrych Thomann: Positionen zur Freiheit des Geistes und zum Individuum im Werk von Paul Valéry. 2001.

Band 6 Eva Leitzke-Ungerer/Andrea Pagni (Hrsg.): Europäische Regionalkulturen im Vergleich. 2002.

Band 7 Hardarik Blühdorn / Jürgen Schmidt-Radefeldt (Hrsg.): Die kleineren Wortarten im Sprachvergleich Deutsch-Portugiesisch. 2003.

Band 8 Eva Leitzke-Ungerer: Frankreichs Regionalkulturen im Französischunterricht. Projekte zur Bretagne, zu Okzitanien, Korsika und zum Elsass. 2004.

Band 9 Norma Esther Díaz: Sprachkontakt in Nôvo Berlim (Rio Grande do Sul). Doppelte Insellage (Eine Fallstudie). 2004.

Band 10 Jürgen Schmidt-Radefeldt (Hrsg.): Portugiesisch kontrastiv gesehen und Anglizismen weltweit. 2006.

Band 11 Paul Ryan: Paul Valéry et le dessin. 2007.

Band 12 Christina Vogel (éd.): Valéry et Léonard: le drame d'une rencontre. Genèse de I'Introduction à la méthode de Léonard de Vinci. 2007.

Band 13 Masahiko Kimura: Le Mythe du Savoir : Naissance et évolution de la pensée scientifique chez Paul Valéry (1880-1920). 2009.

Band 14 Christina Märzhäuser: Portugiesisch und Kabuverdianu in Kontakt. Muster des Codeswitching und lexikalische Innovationen in Raptexten aus Lissabon. 2011.

Band 15 Aurelia Merlan: Sprachkontakt und Sprachenwechsel im portugiesisch-spanischen Grenzgebiet. Das Mirandesische. 2012.

Band 16 Ulrich Hoinkes (Hrsg.): Die kleineren Sprachen in der Romania. Verbreitung, Nutzung und Ausbau. 2013.

Band 17 Aurelia Merlan / Jürgen Schmidt-Radefeldt (Hrsg.): Portugiesisch als Diasystem. O Português como Diassistema. 2013.

www.peterlang.com 